\title{
Resource Management and Contingencies in Aerospace Concurrent Engineering
}

\author{
Gabe Karpati \\ NASA GSFC, Greenbelt, MD 20771 \\ Tupper Hyde \\ NASA GSFC, Greenbelt, MD 20771 \\ Hume Peabody \\ NASA GSFC, Greenbelt, MD 20771 \\ Matthew Garrison \\ NASA GSFC, Greenbelt, MD 20771
}

\begin{abstract}
A significant concern in designing complex systems implementing new technologies is that while knowledge about the system is acquired incrementally, substantial financial commitments, even make-or-break decisions, must be made upfront, essentially in the unknown. One practice that helps in dealing with this dichotomy is the smart embedding of contingencies and margins in the design to serve as buffers against surprises. This issue presents itself in full force in the aerospace industry, where unprecedented systems are formulated and committed to as a matter of routine. As more and more aerospace mission concepts are generated by concurrent design laboratories, it is imperative that such laboratories apply well thought-out contingency and margin structures to their designs.

The first part of this publication provides an overview of resource management techniques and standards used in the aerospace industry. That is followed by a thought provoking treatise on margin policies. The expose presents the actual flight telemetry data recorded by the thermal discipline during several recent NASA Goddard Space Flight Center missions. The margins actually achieved in flight are compared against pre-flight predictions, and the appropriateness and the ramifications of having designed with rigid margins to bounding stacked worst case conditions are assessed.

The second half of the paper examines the particular issues associated with the application of contingencies and margins in the concurrent engineering environment. In closure, a discipline-by-discipline disclosure of the contingency and margin policies in use at the Integrated Design Center at NASA's Goddard Space Flight Center is made.
\end{abstract}

\section{Introduction}

$\mathrm{S}$ pace flight missions are defined by their engineering resources, primarily by their mass, because at the first order resources, especially mass, equal cost in roughly linear fashion. A well-known "urban legend" puts the total lifecycle cost of one kilogram of space flight hardware at one million dollars. This mass-cost correlation is one of the principal reasons why tight management of the engineering resources of space flight missions is so important. A project whose resources are well managed is most likely a project in good shape. Conversely, at times even the cause of mission failures can be traced back to resource management problems.

Besides the mass-cost correlation, the following two reasons also exact smart resource management.

1. Examining the evolution of hundreds of mission concepts formulated in concurrent engineering labs, a common trait stands out: space flight mission design is the art of compounded compounding. A subsystem gets 
heavier because another subsystem got heavier. This vicious circle of growth epitomizes the perfect algorithm for a runaway situation; at times projects are lucky if their mass growth spiral is even convergent at all. With the smart use of margins, the vicious circles of growth can be broken, as growth in one subsystem can be absorbed by the margin in another subsystem rather than further driving the growth spiral.

2. While knowledge about a new system implementing novel technologies is only acquired incrementally, substantial commitments, both financial and programmatic, are made upfront, essentially in the unknown. An efficient approach to dealing with this dichotomy is the embedding of contingencies and margins in the design to serve as buffers against surprises.

\section{Resource Management Techniques And Standards In The Aerospace Industry}

Margins are important. The initial allocation and subsequent control of margins throughout the development of a space system is arguably the most critical item in determining the success of a mission. Especially for satellite systems, the mass margin in intimately tied to other engineering and management goals such as performance, cost, schedule, and risk. As the mass of the object to be launched approaches the throw-mass limit of the launch vehicle, decisions by the development team skew more aggressively toward mass savings at the expense of some combination of performance, cost, schedule, or risk. Not all satellite development programs that are over their mass controls are cancelled, but the converse is usually true...that is, cancelled programs are almost always over their mass controls.

Margins are tricky business. If a project takes on overly conservative margins at the beginning of a program, they may have let some mission performance go unrealized, or put themselves in an early decision to use expensive lightweight materials or risky lightweight technology. But projects that take margins that are too low walk the path of many developments where margins erode before launch and even more costly (or risky) late design trades must be made. The only method known for finding the right balance is experience. The maturity of the design and the "battle scars" of experienced designers and project managers drive the margin metrics. Standards, whether held in a development organization or in some published industry standard, are a primary way this experience is passed from project to project.

Of course, margins apply to many metrics in the development of a space system, not just mass. Power, data storage, data downlink, processing, pointing error, and thermal metrics are all common satellite technical performance measures (TPMs). These TPMs are tracked in regular (usually monthly) progress meetings and can be used by subsystem engineers as well as project management and senior management to keep a finger on the pulse of the development. It is rare that mass or the other TPMs ever go down and it is common that several may rise together in a correlated way as one subsystem's growth drives others.

In order to track TPMs over the development schedule, a Growth Allowance and Depletion Schedule is followed. This allows for larger growth allowances and margins early in development that diminish as the project approaches launch. The lines at which exceedences trigger stricter monitoring or mass reduction are established (sometimes "stair-stepping" and sometimes smooth) with agreed to values at key program milestones. Fig. II-1. shows such a plan for mass. A mass properties control plan (MPCP) is established early in a project's life and describes in detail the formal process for

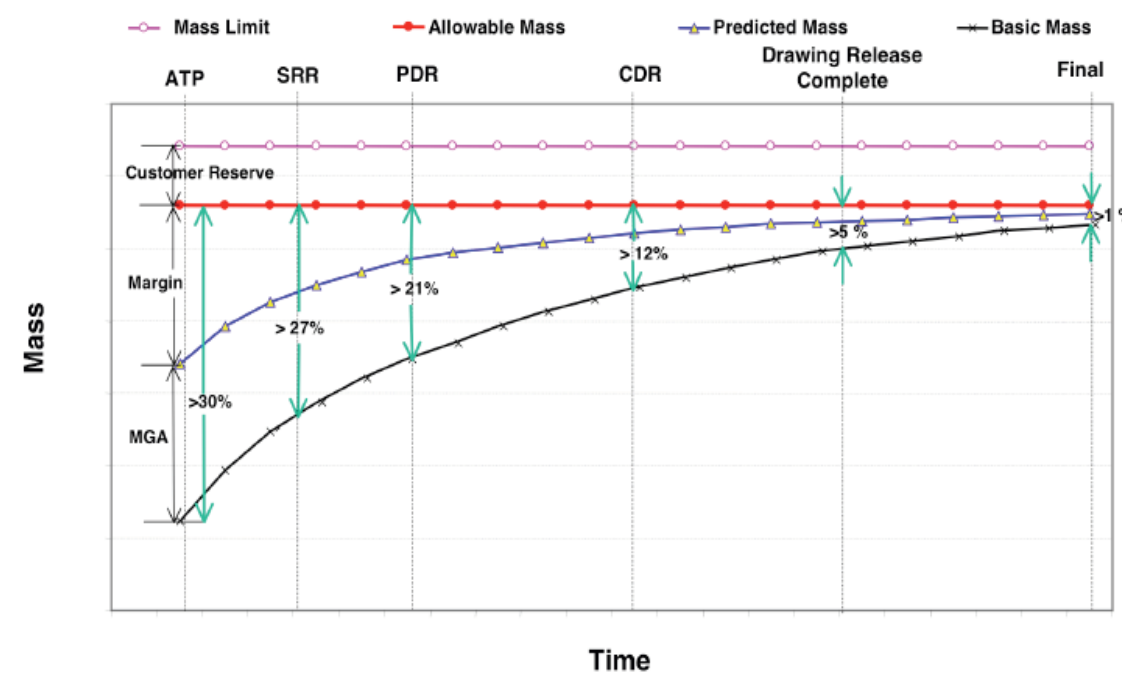

Figure II-1. Contingency and Margin Terminology. (Courtesy AIAA S-1202006.) 
controlling mass. A Mass Control Board (MCB) or other engineering/management board such as a Change Control Board (CCB) performs the function of formally setting the allocations. For example, formal mass threats and opportunities are kept in a database and reviewed periodically. A mass threat has a defined mass growth and a probability of occurrence. If a mass threat is realized, it is formally added to the basic mass estimate and removed from the threat list. Mass threats are usually reviewed concurrently with project risks since risk mitigations are often design changes that result in mass growth. Of course, there can be mass savings opportunities (threats with a negative sign), but those are not realized as often as the threats.

Formal standards for tracking mass margins have existed since the 1970s. The current industry standard for tracking of mass margins on a space system development is AIAA S-120-2006 ${ }^{\mathrm{II}-1}$. This standard, Mass Properties Control for Space Systems combined the earlier MIL-HDBK-1811 (1998) Mass Properties Control for Space systems $^{\text {II-2}}$, MIL-STD-1811 (1992) Mass Properties Control for Space systems ${ }^{\text {II-3 }}$, and MIL-M-38310B (1971) Mass Properties Control Requirements for Missile and Space Systems ${ }^{\mathrm{II}-4}$. AIAA S-120-2006 replaced an ANSI/AIAA standard R-020A-1999 Recommended Practices for Mass Properties Control for Satellites Missiles, and Launch Vehicles $^{\mathrm{II}-5}$. AIAA S-120-2006 is used both as a standard for establishing commonality between programs and contractors, but also is often called out as a contracting document to enforce standard reporting of mass properties to government or commercial customers.

\section{Validation Of Thermal Margin Policies}

This section examines the effects of design and margins rules applied to one subsystem (thermal) in several actually flown space flight missions, and how compliance with those rules impacted system-level resources. It investigates both explicit margins (specified organizational margin requirements) as well as implicit margins (margin hidden in biasing parameters used in stacked worst case analyses). In addition, data is presented that compares those design margins against what was seen in flight in seven recent GSFC missions.

At its most basic level, thermal engineering consists of two activities: sizing radiators to reject the maximum power loads to the hottest flight environment while keeping components below their maximum operating temperature, then sizing heaters to keep the components above their minimum operating or survival temperatures in the coldest environment given the radiator sizes determined above. This process becomes more complex by locating the heat loads farther away from the radiator (requiring heat transport components like heat pipes), needing fine temperature gradient control components, or the need for insulation.

The NASA Goddard Space Flight Center GOLD Rules ${ }^{\mathrm{III}-1}$ specify thermal control system margins that each mission must demonstrate. All of these margin rules are intended to provide excess heating and cooling capability within the thermal control system, so that small failures, last-minute design changes, or on-orbit anomalies will not impact the spacecraft performance. Thermal analysis must show that all components have at least $5^{\circ} \mathrm{C}$ margin on both the hot and cold limits under stacked, worst case conditions. This results in slightly larger radiators, often resulting in more mass, and more orbit-average heater power in cold cases. Additionally, all heater circuits must be sized to a maximum duty cycle of $70 \%$, which provides excess heating capability but increases the current draw by $40 \%$. Finally, all two-phase heat transport systems require 30\% margin on their heat transport capability. These thermal margins are published and generally well-understood by the systems engineering teams.

Less understood are the thermal margins embedded in thermal analysis and design techniques. The thermal analysis cases are usually defined using stacked worst cases, typically varying four biasing parameters: environmental heat loads, coating degradations, component power dissipations and beta angles. These assumptions give the widest possible set of temperature predictions with confidence that the flight cases will always exist within that range assuming normal operations. Little consideration is given to the likelihood or frequency of all these parameters actually occurring simultaneously during the mission as analyzed in a stacked worst case analysis. Since these assumptions all impact the thermal analysis predictions, and the radiator and heater sizes are determined from the analysis, they directly impact the system resources used by the thermal control system.

Furthermore, when reporting predictions, it is common to report the localized spatial nodal temperature extreme at the minimum and maximum points in an orbital analysis. At times, these localized spatial and temporal temperatures may not necessarily be representative of the bulk temperature of a component for which a limit is specified. Lastly, the duration of the mission during which extreme temperatures may be reached should also be considered, since most operational limits are specified based on long term performance over a given temperature range. Short duration excursions outside of this range would likely have a minimal impact on overall mission performance, but it is common for thermal to ensure that $100 \%$ of the mission stays within limits. 
Recent work has assessed the impact of each of biasing parameters on thermal margins. The Global Precipitation Measurement (GPM) mission, being designed, integrated and tested at GSFC, was analyzed using a linear interpolation technique to predict the temperature profile over the entire mission with anchor points based on 10 beta angles, 3 environmental flux, 2 power dissipation, and 2 property configurations for a total of 120 anchor points. The overall profile of the GPM mission was then evaluated to determine what percentage of the mission was spent with a given quantity of margin $^{\text {III-2}}$. This mission estimation technique was validated using flight data from the Lunar Reconnaissance Orbiter mission by comparing predictions for LRO calculated using this approach to actual LRO flight data, and fairly good agreement was found in most circumstances ${ }^{\text {III-3 }}$. The estimation technique was then applied to GPM using an incremental approach for each biasing parameter to determine its impact on the perceived margin compared to the stacked worst cases ${ }^{\mathrm{III}-4}$. Table III-1. shows the impact of progressively incrementing each basing parameter on various critical avionics for the spacecraft.

Previous work has also looked at the impact of the $5^{\circ} \mathrm{C}$ temperature margin on the system resources of mass and power. Four existing radiator designs were used for this study: two that had already been built and two that were in their preliminary design stages. The temperature margin against

Table III-1. Impact of analysis assumptions on worst-case predictions. The impact of multiple stacked worst-case assumptions are shown on the predicted temperature of GPM components. These include the need to meet requirements all the time, using bounding environmental heat loads, worst-case optical property degradations, varying the CBE power and using the worst localized nodal temperature.

\begin{tabular}{|c|c|c|c|c|c|c|c|c|c|c|c|c|}
\hline \multirow[b]{2}{*}{$\begin{array}{c}\text { Mission } \\
\text { Percentile } \\
\end{array}$} & \multicolumn{6}{|c|}{ Decreasing Biasing Parameter } & \multicolumn{6}{|c|}{ Increasing Biasing Parameter } \\
\hline & $5 \%$ & $0 \%$ & $0 \%$ & $0 \%$ & $0 \%$ & $0 \%$ & $95 \%$ & $100 \%$ & $100 \%$ & $100 \%$ & $100 \%$ & $100 \%$ \\
\hline Environment & Avg & Avg & Min & Min & Min & Min & Avg & Avg & Max & Max & Max & Max \\
\hline Properties & $f(t)$ & $f(t)$ & $f(t)$ & BOL & BOL & BOL & $f(t)$ & $f(t)$ & $\mathrm{f}(\mathrm{t})$ & EOL & EOL & EOL \\
\hline $\begin{array}{c}\text { Power } \\
\text { Dissipation } \\
\end{array}$ & Nom & Nom & Nom & Nom & Min & Min & Nom & Nom & Nom & Nom & Max & Max \\
\hline \multirow[t]{2}{*}{\begin{tabular}{c|} 
Reported \\
Temperature
\end{tabular}} & \begin{tabular}{c|}
$\operatorname{Avg}(x)-$ \\
$\operatorname{Min}(t)$
\end{tabular} & $\begin{array}{c}\operatorname{Avg}(\mathrm{x})- \\
\operatorname{Min}(\mathrm{t})\end{array}$ & \begin{tabular}{|c|}
$\operatorname{Avg}(x)-$ \\
$\operatorname{Min}(t)$ \\
\end{tabular} & \begin{tabular}{|c|}
$\operatorname{Avg}(\mathrm{x})-$ \\
$\operatorname{Min}(\mathrm{t})$ \\
\end{tabular} & \begin{tabular}{|c|}
$\operatorname{Avg}(x)-$ \\
$\operatorname{Min}(t)$ \\
\end{tabular} & $\begin{array}{c}\operatorname{Min}(x)- \\
\operatorname{Min}(t)\end{array}$ & $\begin{array}{l}\operatorname{Avg}(x)- \\
\operatorname{Max}(t)\end{array}$ & $\begin{array}{l}\operatorname{Avg}(\mathrm{x})- \\
\operatorname{Max}(\mathrm{t})\end{array}$ & \begin{tabular}{|c|}
$\operatorname{Avg}(\mathrm{x})-$ \\
$\operatorname{Max}(\mathrm{t})$ \\
\end{tabular} & $\begin{array}{l}\operatorname{Avg}(\mathrm{x})- \\
\operatorname{Max}(\mathrm{t})\end{array}$ & \begin{tabular}{|l|}
$\operatorname{Avg}(x)-$ \\
$\operatorname{Max}(t)$
\end{tabular} & $\begin{array}{r}\operatorname{Max}(x)- \\
\operatorname{Max}(t)\end{array}$ \\
\hline & Sum & DT & DT & DT & DT & DT & Sum & DT & DT & DT & DT & DT \\
\hline Component & {$\left[{ }^{\circ} \mathrm{C}\right]$} & {$\left[{ }^{\circ} \mathrm{C}\right]$} & {$\left[{ }^{\circ} \mathrm{C}\right]$} & {$\left[{ }^{\circ} \mathrm{C}\right]$} & {$\left[{ }^{\circ} \mathrm{C}\right]$} & {$\left[{ }^{\circ} \mathrm{C}\right]$} & {$\left[{ }^{\circ} \mathrm{C}\right]$} & {$\left[{ }^{\circ} \mathrm{C}\right]$} & {$\left[{ }^{\circ} \mathrm{C}\right]$} & {$\left[{ }^{\circ} \mathrm{C}\right]$} & {$\left[{ }^{\circ} \mathrm{C}\right]$} & {$\left[{ }^{\circ} \mathrm{C}\right]$} \\
\hline $\mathrm{CDH}^{*}$ & -8.6 & -3.2 & 0.0 & -0.1 & -5.0 & -0.4 & 10.9 & 1.6 & 0.6 & 0.0 & 8.3 & 0.5 \\
\hline MACE* & -8.6 & -3.1 & 0.0 & -0.1 & -5.2 & -0.3 & 11.1 & 1.6 & 0.6 & -0.1 & 8.5 & 0.6 \\
\hline PSE & -11.8 & -3.3 & 0.0 & 0.0 & -8.4 & -0.2 & 13.9 & 1.6 & 0.5 & -0.8 & 11.0 & 1.6 \\
\hline PIE & -10.3 & -4.3 & 0.0 & -0.1 & -5.6 & -0.2 & 11.0 & 1.4 & 0.7 & 0.2 & 8.7 & 0.1 \\
\hline $\mathrm{ST}^{*}$ & -9.6 & -4.0 & 0.0 & -0.2 & -5.3 & -0.1 & 11.1 & 3.1 & 1.0 & 0.3 & 6.7 & 0.2 \\
\hline IRU & -10.8 & -4.0 & 0.0 & -0.2 & -6.2 & -0.4 & 10.9 & 2.8 & 0.8 & 0.0 & 7.0 & 0.4 \\
\hline FEE & -8.5 & -6.1 & 0.0 & -0.3 & -1.6 & -0.4 & 9.1 & 1.3 & 0.8 & -0.1 & 6.7 & 0.3 \\
\hline MTB* & -12.0 & -6.4 & 0.0 & -0.2 & -3.7 & -1.5 & 16.8 & 2.0 & 1.0 & -2.9 & 12.0 & 4.9 \\
\hline RWA5 & -7.3 & -2.1 & 0.0 & 0.0 & -5.0 & -0.2 & 13.4 & 1.7 & 0.8 & -0.1 & 10.6 & 0.4 \\
\hline DFU & -5.9 & -2.1 & 0.0 & -0.1 & -3.5 & -0.2 & 11.6 & 1.2 & 0.7 & 0.0 & 9.5 & 0.1 \\
\hline GPS* & -5.2 & -2.8 & 0.0 & -0.1 & -1.8 & -0.6 & 10.4 & 1.3 & 0.8 & -0.2 & 8.4 & 0.2 \\
\hline RWA1-4* & -7.7 & -4.6 & 0.1 & 0.1 & -2.1 & -1.2 & 13.9 & 1.7 & 1.0 & -0.3 & 10.9 & 0.6 \\
\hline $\mathrm{RF}^{*}$ & -9.0 & -3.2 & -0.1 & 0.0 & -5.7 & 0.0 & 10.3 & 2.0 & 0.7 & 0.3 & 7.3 & 0.0 \\
\hline TAM* & -5.7 & -1.6 & 0.0 & -0.4 & -3.7 & 0.0 & 8.8 & 1.8 & 1.2 & 0.1 & 5.8 & 0.0 \\
\hline Average** & -8.6 & -3.8 & 0.0 & -0.1 & -4.1 & -0.5 & 12.2 & 1.8 & 0.8 & -0.4 & 8.9 & 0.9 \\
\hline
\end{tabular}
the hot operational limits resulted in radiator mass growth of between 0.3 and $0.7 \mathrm{~kg}$ per $100 \mathrm{~W}$ heat load rejected, depending on the radiator views and operating temperatures. Note that this is a first-order impact only; it does not include the impact of heavier structure to support the heavier radiator. Oversizing the radiator also resulted in a survival heater power increase of 4 to $6 \mathrm{~W}$ per $100 \mathrm{~W}$ heat load. These impacts then trickle through other subsystems, increasing the overall capability and complexity of the flight system.

The validation of thermal margin policies, both explicit and implicit, lies in comparison of predicted temperatures to those seen on orbit. Recent work has looked at this comparison for seven recent GSFC missions ${ }^{\text {III-5 }}$. Daily or orbital max temperatures were polled for a total of 209 flight temperature sensors over the entire life of these missions, most of which have operated beyond their designed lifetime. These were then compared against the test-correlated temperature predictions generated before launch. Fig. III-1. shows the results of this study. Across the board, all of the measured flight temperatures were less extreme than the bounding prediction with two 
exceptions: one solar array sensor on a single mission ran warmer than predicted (due to what was later determined to be bad thermal modeling practices on an uncorrelated model), and actuators in general tended to run up to $2^{\circ} \mathrm{C}$ above their stacked worstcase predictions $1 \%$ of the time. The flight data was $2{ }^{\circ} \mathrm{C}$ less than the bounding hot case predictions $95 \%$ of the time, but the average flight data point was $16^{\circ} \mathrm{C}$ lower. Based on these results, it can be concluded that the stacked worst-case assumptions discussed as implicit thermal margin do a good job bounding three standard deviations of all possible orbital thermal environments, but that this may be excess margin for lower-cost more risky missions.

\section{Impacts Of Thermal Margin Policies}

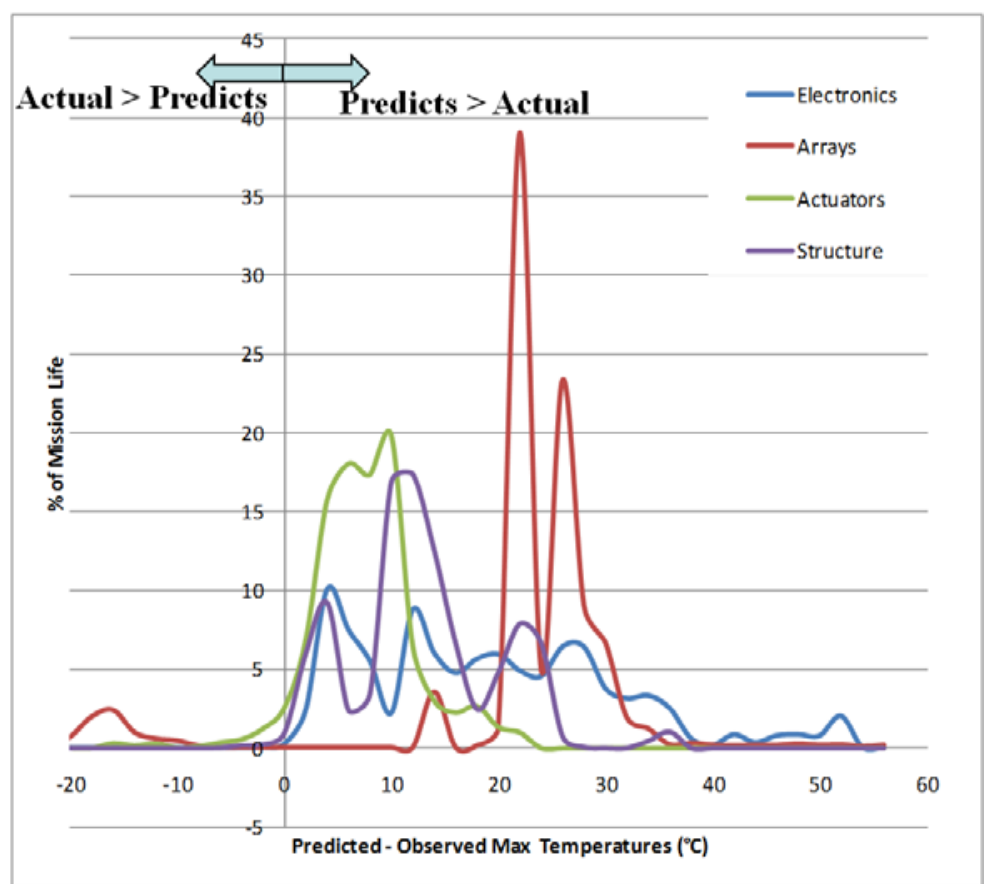

Figure III-1. Flight temperature data compared against

While many missions flown by GSFC have been quite successful, the potential costs associated with designing highly robust spacecraft and predicted temperatures, grouped by component type. All components run below their worst-case hot predictions throughout the mission, with the exception of a single solar array sensor on one mission. instruments that far outlast their design life may have come at the expense of other potential missions. More expensive and highly robust missions consume resources (both money and staffing) that could be alternately used to develop other missions. Ultimately, a choice is presented: more missions with higher risk or fewer missions with lower risk, i. e. how to best maximize the science return on total dollars invested.

The above analyses of the margins held as standard practice by the GSFC Thermal Engineering Branch (both explicit margin required by the organization and implicit margin held as conservatism in the design parameters), suggest that lesser margins could be maintained without adding significant risk. Given that performance limits are generally based on long term operation within the specified limits, maintaining all components within limits for $100 \%$ of the mission with $5^{\circ} \mathrm{C}$ (or greater) margin may be excessive and unnecessary. In fact the typical qualification of the design to temperatures $10^{\circ} \mathrm{C}$ outside of the expected flight temperature extremes itself demonstrates that short duration excursions (i.e. on the order of thermal vacuum testing durations) do not significantly impact the overall performance of the hardware.

Determining the impacts of reduced design margins on a mission's overall cost and schedule is overarching and complex, but the impacts are not inconsequential. The potential of savings in resources may be truly far reaching, as the overly conservative design culture is not limited to thermal engineering, but in fact it is prevalent in almost all disciplines. Significant cost savings could be realized with higher, but manageable, mission risk, by having every subsystem perform overarching analyses to characterize their explicit and implicit margins, and determine the correct margins to be applied for a given risk posture and mission success scenario, rather than - as is the case now apply every time pre-ordered inflexible and overly conservative margins. 


\section{Application of Contingencies and Margins in Concurrent Engineering}

Concurrent engineering at the conceptual design phase must reflect the best guess of what the flight article will look like, and, for all of the reasons stated above, that guess incorporates contingencies and margins. The following two Sections describe how the Mission Design Laboratory (MDL) of the Integrated Design Center (IDC) at NASA Goddard Space Flight Center ${ }^{\mathrm{V}-1,}$ applies contingencies and margins to their designs ${ }^{\mathrm{V}-2}$.

\section{A. Governing Principles}

The governing principles in applying contingencies and margins in the concurrent engineering environment are: Flexibility, Appropriateness, Rightness, and Clarity.

Flexibility is essential in all things concurrent engineering. Considering the extraordinary variety of pre-Phase-A concepts studied in the concurrent design labs of the IDC, from planetary rovers to satellite dispensers, the use of an inflexible set of contingencies and margins with no regard to the unique attributes of each study would be counterproductive. To avoid any rigidity that would lead to an inferior designs, due consideration is given to the unique circumstances of each particular mission. For that one reason: flexibility, the definitions presented here are used only as guidelines in the IDC, not as rules. Compliance is on a "best effort / as practical" basis. The contingency and margin guidelines are also flexible in that they are living documents that evolve as the state of the art in concurrent design advances, and also to reflect changes in applicable industry standards and regulations.

Appropriateness requires applying contingencies and margins at a level that is commensurate with the limited resolution of rapid design. Defining contingencies and margins for concurrent engineering in minute details is not necessary; the resolution must allow easy and rapid application well suited to the quick pace of the work in the labs. While compliance with applicable industry guidelines and standards, such as the GSFC "GOLD Rules"III-1, the Space Mission Analysis and Design handbook ${ }^{\mathrm{V}-3}$, or the GSFC Environmental Verification Standard (GEVS) ${ }^{\mathrm{V}-4}$ is to be maintained, such compliance can be accomplished at higher levels of assembly, for instance by applying contingencies and margins at the subsystem or system levels, as opposed to the line item or component levels.

Rightness requires that the size of contingencies and margins reflect the correct flight-like configuration and not be disproportionate, under or oversized. As contingencies and margins burden the mission concepts with additional cost, mass, volume, power, etc., right sizing them is markedly important for a correct and affordable design. Stacks of contingency pile-ups are to be strictly avoided.

Clarity requires that the contingencies and margins applied be well-defined and easy to understand. Their application should not complicate the study flow by requiring excessive justification or negotiations with the customer. It is appropriate, even expected, that the results of concurrent studies are modified by the customer after leaving the labs; the design sessions themselves often uncover areas needing additional post study attention. If the definition of contingencies and margins that were implemented is clear, then it will be straight-forward for the customers to work with them during the post-study adjustments.

\section{B. Definition of Terms}

Dictionaries define "Contingency" as "a possible occurrence", while "Margin" as "an amount beyond the necessary". Fig. V-1. shows the relationships between the commonly used terms in this field. The most frequently used terms and acronyms, in accordance with standard AIAA S106 ${ }^{\mathrm{II}-1}$, are:

- MPV (Maximum Possible Value)

- MEV (Maximum Expected Value)

- MGA (Maximum Growth Allowance) a.k.a. "Contingency"

- CBE (Current Best Estimate)

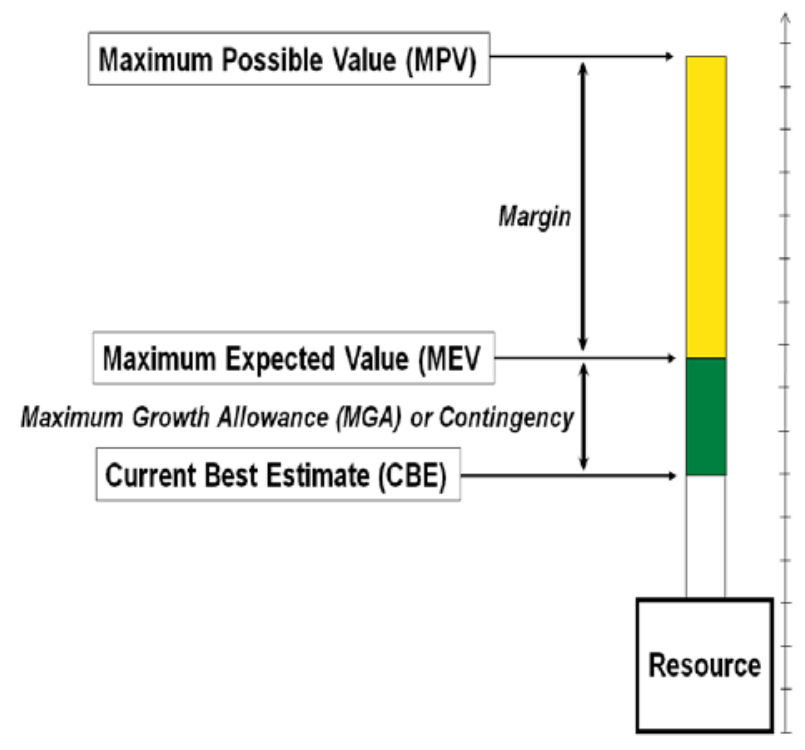

Figure V-1. Contingency and Margin Terminology 
The relationships between the above terms are defined as:

$$
\begin{gathered}
\text { MGA (a.k.a. Contingency) }=(\mathbf{M E V}-\mathbf{C B E}) / \mathbf{C B E} \quad \text { expressed as a } \% \\
\text { Margin }=(\mathbf{M P V}-\mathbf{M E V}) / \mathbf{M E V} \quad \text { expressed as a } \%
\end{gathered}
$$

In common practice, there is much confusion as to which of the two terms on the right side of the above equations is to be placed in the denominator, the "bigger one", or the "smaller one" (correctly it's the smaller one, as shown, yielding more favorable numbers).

Replacing the simplistic term "Contingency" with the two more specific terms "Growth Contingency" (i.e. (MEV - CBE) / CBE), and Resource Contingency (i.e. (MEV - CBE) / MEV) would preempt any such misunderstanding. Likewise, "Margin” could be replaced with the more specific terms "Growth Margin” (i.e. (MPV - MEV) / MEV), and "Resource Margin” (i.e. (MPV - MEV) / MPV). Lastly, to avoid a common cause of confusion, the term "Reserve", should be avoided altogether, as it is ambiguous, confusing, and has been used as a misnomer for a variety of other terms.

\section{Design Based on CBE vs. MEV}

As stated above, a subsystem gets heavier because another subsystem got heavier; subsystem masses drive other subsystem masses. This implies with mathematic clarity that a design sized on MEV values is intrinsically different form a design that used CBE values, even if upon completion an overall MGA was added to the latter. The examples in Figures V-2. and V-3. clearly illustrate this point.

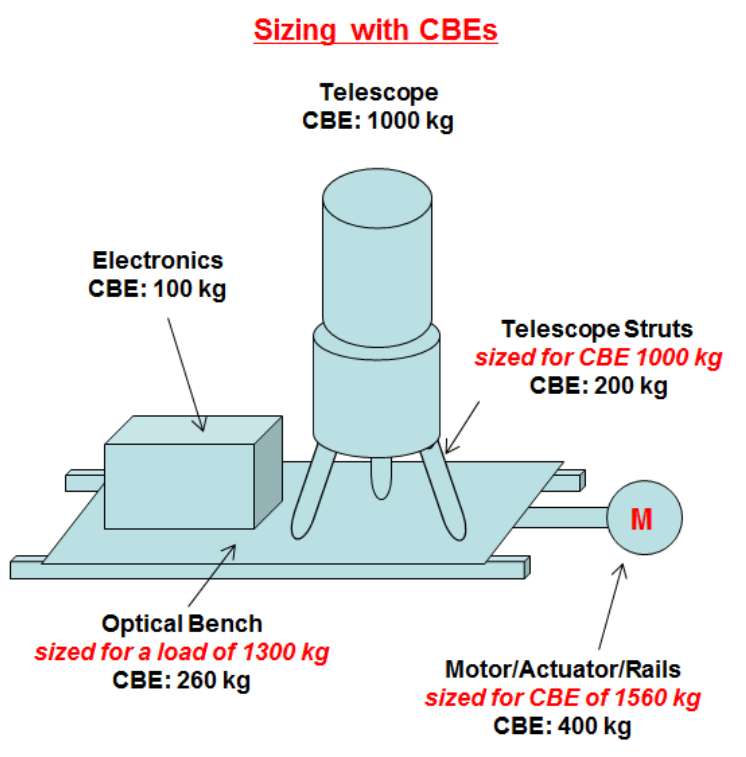

Figure V-2. Example of sizing using CBEs. The total System Mass is CBE $1960 \mathrm{~kg}$. If the Telescope and the Electronics Box weigh in at MEV masses, then the Struts and the Optical bench are undersized!

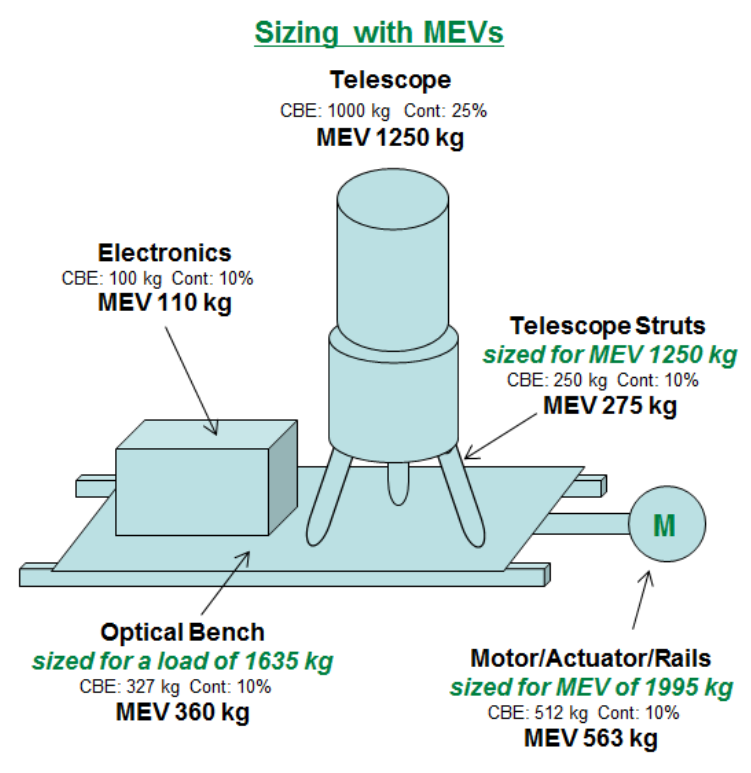

Figure V-3. Example of sizing using MEVs. The total System Mass is CBE $2189 \mathrm{~kg}$, Composite Contingency 17\%, MEV $2558 \mathrm{~kg}$. Even if the Telescope and the Electronics Box weigh in at $M E V$ masses, the Struts and the Optical bench are sized right.

Take a hypothetical telescope with a mass of $1000 \mathrm{~kg}$ CBE (1250 kg MEV), and assume its supporting structure calls for struts that weigh $20 \%$ of the supported mass. The struts sized using the telescope's CBE mass then weigh $200 \mathrm{~kg}$ (CBE) (as shown in Fig. V-2.). A more realistic design (shown in Fig. V-3.) factors in the possible mass 
growth of the telescope by sizing the supporting struts for the telescope's MEV mass of $1250 \mathrm{~kg}$. The same sizing rule (20\% of the supported mass) now call for stronger struts weighing $250 \mathrm{~kg}$ (CBE). In the first design, the mass ratio of the struts (CBE) to the telescope (CBE) was 20\%, but in the second that ratio is $25 \%$. The struts based on CBE masses are undersized, those struts could break. Note, that the "CBE design's" telescope-to-struts mass ratio will never equal that of the "MEV design", not even after applying any overall system level MGA to it; the two designs are really not the same.

Figures V-2. and V-3. clearly illustrate this point, and even take it one level further through the calculations for a moveable platform. At that additional level, the differences between the CBE based and MEV based designs become even more pronounced, the divergence becomes fairly significant.

The "take home message" is that concurrent engineering should generally base its sizing calculations on MEV values. A design based on CBE’s may be wrong.

\section{Contingency Pile-up}

The consecutive allotment of a series of contingencies can have a significant effect on the overall system, as seen in the example in Figures V-2. and V-3. In the Fig. V-3. case, the serial allotment of MGAs was right, but there are many instances when contingency pile-ups are wrong, especially when the contingency pile-ups remain hidden because they cross subsystem or dimension boundaries.

The realistic scenario below from a concurrent study illustrates the nature of hidden contingency pile-up:

- The RF Comm subsystem gets the CBE data rate from Science, and adds $30 \%$ contingency to it.

- With that, RF Comm selects a slightly oversized RF hardware to handle the MEV data rate. Of course, that $R F$ Comm gear is bigger, heavier, and consumes more power, than a comparable hardware sized for CBE data rates would have been.

- $R F$ Comm then sends the (higher) CBE power consumption of the (bigger) RF Comm hardware to the Electrical Power Subsystem (EPS).

- EPS adds its own 30\% contingency to the power consumption of the already oversized box, and sizes a Power System that includes the contingent load of the RF Comm hardware.

- Then, EPS reports not the CBE but the MEV power dissipation of that (bigger) PSE box to Thermal.

- Thermal sizes a radiator for the MEV power dissipation, but in doing so, it adds temperature margins that call for an additionally oversized radiator.

- Mechanical then adds its own contingency the reported radiator mass when sizing the radiator's supporting structures.

- Reaction wheels are then sized to handle that systems MEV inertia plus due GN\&C contingency factored in, which makes the wheels even larger, consuming even more power (reported back to EPS, that adds 30\% contingency to it...)

- ...etc., etc.

Looking back at the data rate as the starting point, it's hard to see how many layers of contingencies were piled on. The bottom line is: concurrent design products are vulnerable to contingency pile-ups, especially so to hidden contingency pile-ups.

The question arises: When is contingency pile-up right, when is it wrong?

Contingency pile-up is right when the causes for the growth of resources over different sequential subsystems or domains are correlated, i.e. one growth drives the other. Consider this example:

- $15 \%$ contingency is added to the CBE mass of a box. As the box could actually grow to that MEV mass, its support structure should be sized for the MEV mass. The MEV based design of the support structure itself then yields a CBE mass for that structure. As that support structure itself could experience mass growth of its own, it is proper to add another contingency \% to its mass, and account for that MEV mass at the System level.

In the above example, the supported mass obviously drove the size of the support structure, the two domains were clearly correlated, thus the consecutive allotment of superimposed contingencies was right.

Contingency pile-up is wrong when the causes for resource growth in different sequential domains in the design are uncorrelated (i.e. one does not drive the other). Take this example:

- $25 \%$ contingency is carried over the CBE mass of an avionics box. The CBE power consumption of the box is 100 W. It does not follow automatically that the Electrical Power Subsystem should size its gear factoring in an MEV power load of $125 \mathrm{~W}$ (incl. 25\% contingency) from that box. Why? Because the power consumption of an avionics box doesn't necessarily grow when its mass grows. Avionics mass growth can be triggered by 
the need for more radiation shielding, or when the exact same electronics circuits don't fit in the box as planned, and a larger housing is needed. Avionics' mass MGA provides cushioning for such mass growth events, neither of which necessarily affects power consumption. This is not to say the Avionics box's power doesn't need contingency; it does. Just that the rationale for sizing that is independent of the rationale for sizing the mass MGA.

In the above example, the two domains were orthogonal, uncorrelated; therefore the automatic consecutive superimposed allotment of contingencies would be wrong. MGAs applied to uncorrelated domains are subject to different rationales, and must be evaluated independently of each other.

General rules are hard to define in this area, logical end-to-end thinking is required to determine the right approach to contingencies across uncorrelated domains.

It is interesting to note that, conversely to contingency, margin doesn't pile up! It is therefore preferable to have a lesser but realistic contingency with the balance carried as margin, than to have unrealistically high contingencies with a lesser margin.

\section{E. Contingency Size}

Too much contingency can stifle a mission while too little can break it. How much contingency is right? Several factors, such as mission class, risk posture, and system resiliency, affect the absolute magnitude of the contingencies and margins that are right for a particular project.

The magnitude of contingencies and margins is a function of the mission class. More contingency is required for a Class A mission then for a Class D mission.

Same goes for mission type. An interplanetary mission, where the launch vehicle's throw mass is exploited to the last ounce, may opt for relatively narrower contingencies and margins, while a LEO mission with throw mass in relative abundance may be more cavalier. Tightening the contingencies and margins is usually compensated by meticulous planning and more aggressive resource management, occasionally coupled by unforgiving "no fly" rules for violators.

An interesting approach in reducing the overall mission level contingencies and margins is enlisting the help of statistical averaging: pairing up subsystems begging for more resources with those having them in excess. On Cassini, a stock market-like resource exchange ${ }^{\mathrm{V}-5}$ was created to that effect, where payload contingencies could be traded according to free market principles.

The magnitude of contingencies and margins is also function of the project's risk posture: obviously less contingency means higher risk; risk of any kind: performance, technology, programmatic, financial, etc.

\section{F. System Resiliency}

The right size of the contingency required in each domain is also a strong function of the resiliency of system performance to the resource growth impacting that domain.

In some cases, exceeding the MEV results in nothing more than a graceful degradation of performance. In such cases lesser contingency is acceptable.

- Slew and repointing performance in many missions exhibits graceful degradation characteristics. If due to mass growth the observatory's inertia exceeds the maximum expected value for which the reaction wheels were sized, the consequence is a proportional increase in slew times, resulting in nothing more serious than a slight degradation of the mission's observing efficiency.

In some other instances, exceeding the MEV causes unacceptable harm to the mission, and is to be avoided at all cost. Obviously, in such cases more generous contingencies are required.

- Mass growth often faces a hard breakpoint, the launch vehicle's throw mass capability to the desired orbit. If the mission's launch mass exceeds the throw mass capability then the target orbit can't be reached; the mission may be over before it began!

In summary, less contingency is needed for phenomena or system performance that exhibits soft or graceful degradation, and more when a hard breakpoint is faced. 
Moments of inertias are calculated from MEV masses, rather than calculated from CBE masses subsequently burdened with contingency, which may produce a false result.

Torque margins are sized per GEVS ${ }^{\mathrm{V}-4}$ as follows: known torque factor of safety: 2.0; variable torque factor of safety: 4.0. These factors of safety apply to the MEV values of all mechanical functions, those driven by motors as well as springs, etc. at BOL, and include all flight drive electronics effects and limitations.

The following factors of safety apply to MEV limit loads in accordance with GEVS ${ }^{\mathrm{V}-4}$, to provide confidence that the hardware will not experience failure or detrimental permanent deformation under test, ground handling, launch, or operational conditions:

\begin{tabular}{lllc}
\multicolumn{1}{c}{ Type } & Static & Sine & Random/Acoustic \\
\hline Metallic Yield & 1.25 & 1.25 & 1.6 \\
Metallic Ultimate & 1.4 & 1.4 & 1.8 \\
Stability Ultimate & 1.4 & 1.4 & 1.8 \\
Beryllium Yield & 1.4 & 1.4 & 1.8 \\
Beryllium Ultimate & 1.6 & 1.6 & 2.0 \\
Composite Ultimate & 1.5 & 1.5 & 1.9 \\
Bonded Inserts/Joints Ultimate & 1.5 & 1.5 & 1.9
\end{tabular}

\section{Contingency and Margin Guidelines for Thermal}

Thermal design is sized to provide a margin between the stacked worst-case flight predictions and component allowable flight temperature limits, both in normal operations and planned contingency modes, in accordance with GEVS $^{\mathrm{V}-4}$ and the applicable GSFC procedures and guidelines for thermal design. The thermal system is sized using MEV power dissipation values to hold a minimum of $10^{\circ} \mathrm{C}$ temperature margin in the radiator sizing calculation. (Note that the actual requirement in GOLD Rules is to have 5C of margin against operational limits.) No other contingency is included.

- As an example, holding a $10^{\circ} \mathrm{C}$ margin at a radiator temperature of $283^{\circ} \mathrm{Kwould}$ result in a radiator size margin of about $15 \%$.

$3 \sigma$ values are used for uncertainties in coatings properties, solar constant, albedo, insulation properties, etc. (The 10 degrees margin above is in addition to the padding provided by using $3 \sigma$ values.)

Radiator sizing must factor in not only power dissipation, but also other contributing factors, such as temperature drop caused by the heat transport device (heat pipes, loop heat pipes, thermal straps, etc.), environmental flux absorbed, absorptance and emittance of thermal coating, orbital modeling (beta angle, sun angle, eclipse), back load from solar arrays, view factor to other instruments or spacecraft components, parasitic heat load (major factor for detectors passively cooled at cryogenic temperatures), etc.

Survival heaters are to be sized to deliver the nominal CBE power at 70\% duty cycle, which means that the heater circuit is capable of a $40 \%$ increase in power.

\section{E. Contingency and Margin Guidelines for Cryogenics}

$100 \%$ contingency is applied to the cryogenic system's heat lift capability (i.e. the actual cryogenic temperature CBE heat loads are doubled for sizing purposes).

Note: The National Institute of Standards and Technology (NIST) considers the field of cryogenics as that involving temperatures below $-180^{\circ} \mathrm{C}$.

\section{F. Contingency and Margin Guidelines for Flight Dynamics}

Flight Dynamics and propellant calculations are more analytical and more predictable than the products of most other disciplines. The IDC margin policies reflect that.

Flight Dynamics reports $3 \sigma$ delta-v's values, based on:

- $3 \sigma$ launch vehicle delta-v dispersion.

- $2 \sigma$ solar cycle predicts. 
Flight Dynamics generates two mission total CBE delta-v's rackups:

- One for the required mission life.

- One for the "goal" mission life.

The published $3 \sigma$ worst case CBE delta-v numbers don't contain any contingency, except in rare exceptional cases where no reliable accurate delta-v calculations can be made.

\section{G. Contingency and Margin Guidelines for Propulsion}

Below is a tabulation of the main items defining propulsion system sizing, with an item by item listing of how all the contributors to that item's sizing are to be factored in.

- Delta-v: Use 3 $\sigma$ worst case CBE delta-v numbers as reported by Flight Dynamics, without any additional contingency. Size the propellants for the required mission life delta-v (not the "goal" mission life delta-v).

- Propelled mass: Calculate propellants using MEV masses.

- Specific impulse: Use realistic $\mathrm{I}_{\mathrm{sp}}$ 's substantiated by component data sheets and burn modes. Factor in $\mathrm{I}_{\mathrm{sp}}$, and "thrust vs. pressure" functions for blow-down systems. Use $2 \%$ to $5 \%$ margins on $\mathrm{I}_{\mathrm{sp}}$, and thrust for heritage COTS units. When thruster data is unavailable, a conservative $\mathrm{I}_{\mathrm{sp}}$ is to be factored in, $10 \%$ below typical nominal values.

- Other factors: Add thruster cosine losses as applicable. For non-impulsive maneuvers, take into account the efficiency of long burn times and also thrust vector orientation. Considers worst case propellant loading temperatures and density variations.

- $\quad$ Non-thrust propellant use: Account for the MEV value of any other propellant use during the mission, such as reaction wheel offloads, propulsive slews, stray torque compensation, etc.

- "Propellant Taxes": Add $5 \%$ ACS Tax on all delta-v numbers. Add 3\% to 5\% for ullage and residuals on total propellant, dependent on manifold size, complexity, and tank expulsion efficiency.

- Tank sizing: Size tanks for a wet launch mass that equals the launch vehicle throw mass, with all the above considerations factored in.

Sizing is to be verified by "bottoms up" calculations. When the sizing is complete, report the MEV masses and dimensions of the propulsion hardware to Systems.

\section{H. Contingency and Margin Guidelines for Attitude Control}

Below is a tabulation of the main items defining the sizing of the Attitude Control system, with an item by item listing of how all the contributors to that item's sizing are to be taken into account.

- Mass properties: The ACS is sized to MEV mass properties.

- Actuators: Use MEV mass properties and MEV moments of inertia in sizing of the actuators (typically reaction wheels, magnetic torquers, and thrusters).

- Disturbances: Apply variable contingencies, typically $100 \%$ for environmental and stochastical disturbances.

- Torques: Apply 30 - 50\% contingency on slew torques and momentum unloading torques.

- Performance modeling: Use $3 \sigma$ uncertainties in reaction wheel, thruster, torquer, etc. performance modeling, and then add a low contingency (5 to $10 \%$ ), whose value depends on what the mission can bear and what's the degradation posture (soft vs. hard). Factor in variable contingencies for slew times, as applicable.

- Knowledge and control requirements: Typically $40 \%$ contingency is applied to the knowledge and control requirements to provide padding for factors outside of the ACS (such as thermal distortion, instrument misalignments, instrument jitter, orbit error (for geolocation missions), etc.

- Controller Stability Margins: apply at least $6 \mathrm{~dB}$ for rigid body stability; 30 degrees phase margin; $12 \mathrm{~dB}$ gains margin. In practice, the high-level controller design specified in the MDL does not permit precise determination of these margins; instead ROM values are produced that satisfy this requirements.

\section{Contingency and Margin Guidelines for RF Communications}

Below is a tabulation of the main items defining the sizing of the RF Communications system, with an item by item listing of how all the contributors to that item's sizing are to be taken into account.

- Data rates: Apply 30\% contingency on CBE data rates.

- Components: All RF components sized to MEV values. 
- Communication links: Size to $3 \mathrm{~dB}$ margin, except for TDRSS links, where $0 \mathrm{~dB}$ margin is acceptable.

- Antenna coverage: Assume 90-99\%, with the assumption clearly stated.

- Rain attenuation: Assume 90-99\%, with the assumption clearly stated.

\section{J. Contingency and Margin Guidelines for Flight Software / IT}

On all flight software items, such as ROM and PROM sizes, CPU usage, various bus loading factors, timing margins, etc., always apply $50 \%$ contingency across the board, except as stated below:

- PCI Bus: 75\% contingency required.

- 1553 Bus: 30\% contingency required.

\section{K. Contingency and Margin Guidelines for Radiation}

The Total Ionizing Dose (TID) values published by the Radiation discipline are typically MEV values in that they typically include a 2x Radiation Design Margin (RDM) (i.e. the contingency applied is $100 \%$ ). The fact that the TID numbers include a " $2 x \mathrm{RDM}$ " must be clearly noted.

Component single event specification: no SEE may cause permanent damage to a system or subsystem. Electronic components shall be designed to be immune to SEE-induced performance anomalies or outages that require groundintervention to correct. If a device is not immune to SEEs, analysis for SEE rates and effects must take place based on the linear energy transfer threshold (LETth) of the candidate devices as shown in Table

Table VI-2. Linear energy transfer thresholds. Environment to be assesses as shown.

\begin{tabular}{ll|}
\hline Device Threshold & Environment to be Assessed \\
\hline $\mathrm{LET}_{\text {th }}<20\left(\mathrm{MeV} \cdot \mathrm{cm}^{2}\right) / \mathrm{mg}$ & $\mathrm{GCR}$, trapped protons, SPE \\
\hline $\mathrm{LET}_{\text {th }}=20-75\left(\mathrm{MeV} \cdot \mathrm{cm}^{2}\right) / \mathrm{mg}$ & $\mathrm{GCR}, \mathrm{SPE}$ \\
\hline $\mathrm{LET}_{\text {th }}>75\left(\mathrm{MeV} \cdot \mathrm{cm}^{2}\right) / \mathrm{mg}$ & No analysis required \\
\hline
\end{tabular}
VI-2.

\section{Contingency and Margin Guidelines for Schedule}

The funded schedule reserves to be factored in the project schedule and cost calculations are:

- 1 month per year in Phase C and Phase D1 (build).

- 2 months per year in Phase D2 (observatory I\&T).

- 3 months per year in Phase D3 (launch site ops).

\section{Conclusions}

This paper touched on a number of areas associated with the application of contingencies and margins during the formulation phases of space flight missions.

Section II. presented a survey of resource management techniques and standards used across the aerospace industry.

In Sections III. and IV., the impacts of various parameters on thermal margins were investigated, and the margins actually achieved in flight in several recently flown NASA Goddard Space Flight Center missions were compared against pre-flight predictions. As practically all components maintained excess margins at all times, the findings suggest that lesser margins could have been applied without adding significant risk to those missions. The ramifications of lesser contingencies and margins could be far reaching. While most missions flown by GSFC using the present conservative margin policies have been quite successful, the costs associated with designing highly robust spacecraft and instruments that far outlast their design life come at the expense of other potential missions. More expensive highly robust missions consume resources that could be alternately used to develop other potential missions. Ultimately, the choice is presented: more missions with higher risk or fewer missions with lower risk; i.e. how to best maximize the science return on the total dollars invested.

Section V. examined the particular issues associated with the application of contingencies and margins in the concurrent engineering environment, and Section VI. provided a detailed disclosure of the contingency and margin policies in effect at the Integrated Design Center at NASA’s Goddard Space Flight Center.

13

Resource Management and Contingencies in Aerospace Concurrent Engineering 


\section{Acknowledgements}

The IDC Contingency and Margin Guidelines were formulated with advice and contributions by the following Goddard Space Flight Center employees: Rick Wesenberg, Anel Flores, Bruce Campbell, Dave DiPietro, Lee Niemeyer, Ted Swanson, Robin Mauk, John Oberright, Mark Steiner, Eric Stoneking, Bob Beaman, Mike Choi, Wes Ousley, Kequan Luu, Louise Bashar, Terry Smith, all from GSFC Code 500, Phil Kalmanson from Orbital Sciences Corp., and by many other IDC Discipline Engineers.

\section{References}

${ }^{\text {II-1 }}$ AIAA Standard, Mass Properties Control for Space Systems, S-120-2006e, 2006.

${ }^{\text {II-2 }}$ MIL-HDBK-1811: Department Of Defense Handbook, Mass Properties Control for Space Systems, 12 August 1998

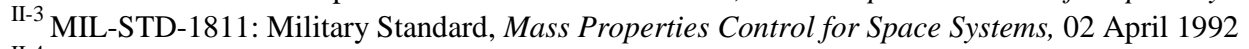

${ }^{\mathrm{II}-4}$ MIL-M-38310B: Mass Properties Control Requirements for Missile and Space Systems, 1 July 1971.

II-5 AIAA Standard, Military Standard, Recommended Practices for Mass Properties Control for Satellites Missiles, and Launch Vehicles, ANSI/AIAA R-020A-1999

III-1 NASA Goddard Technical Standard GSFC-STD-1000E, Goddard Space Flight Center Rules for the Design, Development, Verification and Operation of Flight Systems, (GOLD Rules), 2009.

III-2 Rodriguez J., Peabody H., Benitez V., "Thermal Margin Assessment of the Global Precipitation Measurement Satellite (GPM)“, Spacecraft Thermal Control Workshop 2012.

${ }^{\text {III-3 }}$ Garrison, M., Peabody H., Peabody S., "Mission Life Thermal Analysis and Environment Correlation for the Lunar Reconnaissance Orbiter”, International Conference on Environmental Systems 2012.

III-4 Rodriguez J., Peabody H., Benitez V., “Thermal Margin Study for the Global Precipitation Measurement Spacecraft”, International Conference on Environmental Systems 2012.

III-5 Garrison, M., "Statistical Analysis of Thermal Analysis Margins”; Thermal and Fluids Analysis Workshop $2011 ; 2011$.

${ }^{\mathrm{V}-1}$ Karpati, G., Martin, J., Steiner, M., Reinhardt, K., "The Integrated Mission Design Center (IMDC) at NASA Goddard Space Flight Center”; IEEE Proceedings, Volume 8, Issue , Pages: 8_3657 - 8_3667, 2003.

V-2 Karpati, G., Polacek, G., Avnet, M., Panek, J., Campbell, B.; “Information Exchange In a Concurrent Engineering Lab, and The Tools That Enable It”; AIAA Space 2011 Proceedings; 2011.

${ }^{\mathrm{V}-3}$ Wertz, J.R., Space Mission Analysis and Design, 3rd ed., eds. J.R. Wertz and W.J. Larson, . El Segundo, CA, Microcosm, 1999.

V-4 Goddard Technical Standard, Goddard Technical Standard, General Environmental Verification Standard (GEVS) for GSFC Flight Programs And Projects, GSFC-STD-7000

${ }^{V-5}$ Wessen, Randii R., Porter, David, Evaluation of the Cassini resource exchange; SPIE Proceedings, Vol. 2803, Pages: 234$244 ; 1996$.

\section{Biographical Information}

Gabe Karpati is the Senior Systems Engineer of the Integrated Design Center at the Goddard Space Flight Center. At NASA since 1985, Mr. Karpati focuses primarily on space flight mission architecture and concurrent engineering. He has participated in various capacities in a number of missions, including SSBUV, COBE, Hubble Space Telescope, WIND, Polar, Cassini, and Spitzer; and has led the formulation of over a hundred mission concepts, including the Altair Manned Lunar Lander, the Orion Crew Exploration Vehicle, and astrophysical and planetary studies commissioned by the National Academy of Science. Mr. Karpati holds Masters in Electrical/Mechanical Engineering from the Technical University of Budapest. He lives in Bethesda, MD, with his wife and three children.

Dr. Tupper Hyde is the Associate Chief of the Mission Engineering and Systems Analysis Division at NASA Goddard Spaceflight Center. His specialties include precision pointing, vibration control, systems engineering, and technology development planning and management. He serves as the technologist for Advanced Technology Large Aperture Space Telescope (ATLAST), a proposed next generation observatory to HST and JWST. He received his BS from MIT (1988) in Aeronautics and Astronautics, his MS from Stanford University (1989) in space robotics, and his PhD from MIT (1995) in controls and estimation. He worked for Honeywell Space Systems doing attitude and vibration control for commercial, civil, military, and special satellite programs before joining NASA in 2002 where he was the controls lead for JWST and the Mission Systems Engineer for LISA before becoming the Chief 
Technologist for AETD in 2007. Tupper is a Lieutenant Colonel in the US Army Reserve and enjoys boating and geocaching with his family in Severna Park, Maryland.

Hume Peabody is a Staff Engineer in the Thermal Engineering Branch as Goddard Space Flight Center. He received Bachelor of Science and Master of Science degrees from Virginia Tech in Mechanical Engineering before joining Swales Aerospace in 1998. During his 9 year tenure at Swales, he provided thermal support for numerous projects including EOS, MetOp, LISA, and HST. In 2007, he joined the Thermal Engineering Branch at the Goddard Space Flight Center where he has supported numerous programs including GPM, TIRS, and JWST. He has authored numerous papers throughout the years as well as performing paper reviews for the ICES and TFAWS conferences.

Matt Garrison is a thermal engineer at the NASA Goddard Space Flight Center. He joined NASA in 2005 and has served as a thermal engineer on the James Webb Space Telescope, Lunar Reconnaissance Orbiter, Global Precipitation Measurement and Ice, Cloud and Elevation Satellite II missions. He received his Bachelors of Science and Masters of Science in Aerospace Engineering from Penn State University. 